\title{
SINGULAR QUASILINEAR ELLIPTIC SYSTEMS WITH GRADIENT DEPENDENCE
}

\author{
HALIMA DELLOUCHE AND ABDELKRIM MOUSSAOUI*
}

\begin{abstract}
In this paper, we prove existence and regularity of positive solutions for singular quasilinear elliptic systems involving gradient terms. Our approach is based on comparison properties, a priori estimates and Schauder's fixed point theorem.
\end{abstract}

\section{INTRODUCTION}

We deal with the following quasilinear elliptic system

$$
(\mathrm{P}) \quad\left\{\begin{array}{l}
-\Delta_{p_{1}} u=f_{1}(x, u, v, \nabla u, \nabla v) \text { in } \Omega \\
-\Delta_{p_{2}} v=f_{2}(x, u, v, \nabla u, \nabla v) \text { in } \Omega \\
u, v>0 \quad \text { in } \Omega \\
u, v=0 \quad \text { on } \partial \Omega
\end{array}\right.
$$

where $\Omega \subset \mathbb{R}^{N}(N \geq 2)$ is a bounded domain with smooth boundary $\partial \Omega$ and $\Delta_{p_{i}}$ stands for the $p_{i}$-Laplacian differential operator on $W_{0}^{1, p_{i}}(\Omega)$ with $1<p_{i} \leq N, i=1,2$. A solution of $(\mathrm{P})$ is understood in the weak sense, that is a pair $(u, v) \in W_{0}^{1, p_{1}}(\Omega) \times W_{0}^{1, p_{2}}(\Omega)$ with $u, v$ positive a.e. in $\Omega$, and satisfying

$$
\begin{aligned}
& \int_{\Omega}|\nabla u|^{p_{1}-2} \nabla u \nabla \varphi d x=\int_{\Omega} f_{1}(x, u, v, \nabla u, \nabla v) \varphi d x, \\
& \int_{\Omega}|\nabla v|^{p_{2}-2} \nabla v \nabla \psi d x=\int_{\Omega} f_{2}(x, u, v, \nabla u, \nabla v) \psi d x,
\end{aligned}
$$

for all $(\varphi, \psi) \in W_{0}^{1, p_{1}}(\Omega) \times W_{0}^{1, p_{2}}(\Omega)$. The nonlinear terms $f_{i}: \Omega \times(0,+\infty)^{2} \times$ $\mathbb{R}^{2 N} \rightarrow(0,+\infty), i=1,2$, are Carathéodory functions, that is, $f_{i}\left(\cdot, s_{1}, s_{2}, \xi_{1}, \xi_{2}\right)$ is measurable for every $\left(s_{1}, s_{2}, \xi_{1}, \xi_{2}\right) \in(0,+\infty)^{2} \times \mathbb{R}^{2 N}$ and $f_{i}(x, \cdot, \cdot, \cdot, \cdot)$ is continuous for a.e. $x \in \Omega$.

Observe that the dependence on the gradient of the nonlinearities $f_{1}$ and $f_{2}$ deprives system $(\mathrm{P})$ of a variational structure thereby making it impossible applying variational methods. Moreover, due to such convection terms, even the application of the so-called topological methods as sub-super solutions and fixed points technics is not standard. Another important aspect of problem $(\mathrm{P})$ is that the reaction-convection term $f_{i}(x, u, v, \nabla u, \nabla v)$, which is expressed with the solution and its gradient, can exhibit singularities when the variables $u$ and $v$ approach zero. This occur under the following growth

Key words and phrases. p-laplacian; singular systems; gradient estimate, regularity, convection terms; fixed point.

* Corresponding author. 
assumption on functions $f_{1}$ and $f_{2}$, which will be referred throughout the paper as $\left(\mathrm{H}_{f}\right)$ :

$\left(\mathrm{H}_{f}\right)$ : There exist constants $M_{i}, m_{i}>0, \gamma_{i}, \theta_{i} \geq 0, r_{i}>N$ and $\alpha_{i}, \beta_{i} \in$ $\mathbb{R}$ such that

$$
m_{i} s_{1}^{\alpha_{i}} s_{2}^{\beta_{i}} \leq f_{i}\left(x, s_{1}, s_{2}, \xi_{1}, \xi_{2}\right) \leq M_{i} s_{1}^{\alpha_{i}} s_{2}^{\beta_{i}}+\left|\xi_{1}\right|^{\gamma_{i}}+\left|\xi_{2}\right|^{\theta_{i}}
$$

for a.e. $x \in \Omega$, for all $s_{1}, s_{2}>0$, and all $\xi_{1}, \xi_{2} \in \mathbb{R}^{N}$, with

$$
-1 / r_{i} \leq \alpha_{i}+\beta_{i}<\frac{p_{i}-1}{r_{i}} \text { and } \max \left\{\gamma_{i}, \theta_{i}\right\}<\frac{p_{i}-1}{r_{i}} \text {, for } i=1,2 \text {. }
$$

Quasilinear convective problem $(\mathrm{P})$ is involved in various nonlinear processes that occur in many engineering and natural systems. In biology and physiology, it arises in heat transfer of gas and liquid flow in plants and animals as well as in cell reactors, incubators, and biomass systems. In chemical processes, it appears in catalytic and noncatalytic reactions in exothermic and endothermic reacting flows and in polymer processing. In geology, it is involved in thermoconvective motion of magmas and in melt solidification in magma chambers and during volcanic eruptions. It also arises in global climate energy balance models by coupling the equation for the mean surface temperature on $\partial \Omega$ with the equation in $\Omega$ for the ocean temperature. For more inquiries on modeling physical phenomena we refer to [15, 8] and the references therein. Furthermore, many important singular systems could be incorporated in the statement of $(\mathrm{P})$. In this respect, we mention for instance Gierer-Meinhardt system which models some biochemical processes 17, as well as singular Lane-Emden system which arises in astrophysics [13.

Nevertheless, despite its obvious importance, quasilinear singular convective system $(\mathrm{P})$ has been rarely investigated in the literature. Actually, in 6], problem $(\mathrm{P})$ subjected to growth condition like $\left(\mathrm{H}_{f}\right)$ with

$$
\alpha_{1}, \beta_{2}<0<\alpha_{2}, \beta_{1}
$$

was examined under cooperative structure of nonlinearities $f_{1}(x, u, v, \nabla u, \nabla v)$ and $f_{2}(x, u, v, \nabla u, \nabla v)$. This means that the latter are increasing with respect to $v$ and $u$, respectively. The complementary situation with respect to (1.3) is the so-called competitive system. In this context, singular system $(\mathrm{P})$ is studied in [23], under some appropriate growth conditions that does not fit our setting in $\left(\mathrm{H}_{f}\right)$, where the singularities come out through the solution and its gradient. It is worth pointing out in the previous papers that a control on the gradient of solutions, besides the one on solutions, is required hence the use of [5, Lemma 1]. At this point, in [23, the gradient estimate is obtained through some specific growth conditions combined with properties of the eigenfunction corresponding to the first eigenvalue of the operator $-\Delta_{p_{i}}$, while in [6], besides (1.3), it is requested that $\alpha_{i}+\beta_{i} \geq 0$, for $i=1,2$, which is the key ingredient so that [5, Lemma 1] could be applicable. 
Unlike quasilinear systems, meaningful contributions are already available for semilinear singular convective systems (i.e., $p_{1}=p_{2}=2$ ). Here, we quote for instance [2, 3] and the references therein, where the linearity of the principal part has been crucial. The particular case in singular system (P) when the convection terms $\nabla u$ and $\nabla v$ are removed has received a special attention. Relevant contributions regarding this topic can be found in [1, 9, 10, 11, 16, 20, 21, 22] and the references given there.

Motivated by the aforementioned papers, the aim of this work is to establish existence and regularity of (positive) solutions for quasilinear singular convective system $(\mathrm{P})$ subjected to the growth condition $\left(\mathrm{H}_{f}\right)$. The main result is formulated as follows.

Theorem 1.1. Under assumption $\left(\mathrm{H}_{f}\right)$, system $(\mathrm{P})$ admits a positive solution $(u, v)$ in $C_{0}^{1, \sigma}(\bar{\Omega}) \times C_{0}^{1, \sigma}(\bar{\Omega})$ for certain $\sigma \in(0,1)$.

The main technical difficulty consists in the involvement of gradient terms in system $(\mathrm{P})$ as well as the presence of singular terms that can occur under hypothesis $\left(\mathrm{H}_{f}\right)$. This difficulty is heightened by the very marked singularity character of $(\mathrm{P})$ that arises from $\left(\mathrm{H}_{f}\right)$ when $\alpha_{i}+\beta_{i}<0$ for $i=1,2$. Moreover, unlike in the earlier papers, neither cooperative nor competitive structure on the system is imposed. In fact, these both complementary structures for the system $(\mathrm{P})$ are handled simultaneously without referring to them despite their important structural disparity that makes functions $f_{1}$ and $f_{2}$ behaving in a drastically different way.

Our approach is chiefly based on Schauder's fixed point Theorem (see 25). We establish some comparison arguments through suitable functions with an adjustment of adequate constants. These functions beget a positive rectangle providing a localization of an eventual solution. On the other hand, we provide a priori estimate on the gradient of solutions by exploiting the growth condition $\left(\mathrm{H}_{f}\right)$ together with [7, Theorem 3.1; Remark 3.3]. Thence, this established a control on solutions and their gradient that combined together leads to a fixed point through Schauder's Theorem, which is actually a solution of $(\mathrm{P})$. It is worth pointing out that our argument is substantially different from that developed in [6] where extremal solutions concept is relevant. Another significant feature of our existence result concerns the regularity part. At this point, Theorem 2.1 stated in section 2 provides a regularity result for problem (P) extending [12, Lemma 3.1] to singular systems involving convection terms.

The rest of the paper is organized as follows. Section 2 establishes gradient estimates and regularity result; Section 3 deals with comparison properties; Section 3.4 contains the proof of the main result.

\section{Gradient estimate And Regularity}

In the sequel, the Banach spaces $W^{1, p}(\Omega)$ and $L^{p}(\Omega)$ are equipped with the usual norms $\|\cdot\|_{1, p}$ and $\|\cdot\|_{p}$, respectively, whereas the space $W_{0}^{1, p}(\Omega)$ 
is endowed with the equivalent norm $\|u\|=\left(\int_{\Omega}|\nabla u|^{p} \mathrm{~d} x\right)^{\frac{1}{p}}$. We denote by $p^{\prime}=\frac{p}{p-1}$. We also utilize $C^{1}(\bar{\Omega})$ and $C_{0}^{1, \sigma}(\bar{\Omega})$ such that for $\sigma \in(0,1)$ we have $C_{0}^{1, \sigma}(\bar{\Omega})=\left\{u \in C^{1, \sigma}(\bar{\Omega}): u=0\right.$ on $\left.\partial \Omega\right\}$. The continuity of the embedding $W_{0}^{1, p}(\Omega)$ in $L^{r}(\Omega)$, for $1 \leq r \leq \frac{N p}{N-p}$, guarantees the existence of a constant $c_{r}>0$ such that

$$
\|u\|_{r} \leq c_{r}\|u\| \text { for all } u \in W_{0}^{1, p}(\Omega) .
$$

Let us introduce the quasilinear system

$$
\left(\mathrm{P}_{h}\right) \quad\left\{\begin{array}{l}
-\Delta_{p_{1}} u=h_{1}(x, u, v, \nabla u, \nabla v) \text { in } \Omega \\
-\Delta_{p_{2}} v=h_{2}(x, u, v, \nabla u, \nabla v) \text { in } \Omega \\
u, v=0 \quad \text { on } \partial \Omega,
\end{array}\right.
$$

where $h_{i}: \Omega \times \mathbb{R}^{2} \times \mathbb{R}^{2 N} \rightarrow \mathbb{R}$ is a Carathéodory function satisfying the following assumption:

$\left(\mathrm{H}_{h}\right)$ : There exist constants $M>0, r_{i}>N$ and $\mu_{i}<0 \leq \gamma_{i}, \theta_{i}$ such that

$$
\max _{i=1,2}\left|h_{i}\left(x, s_{1}, s_{2}, \xi_{1}, \xi_{2}\right)\right| \leq M\left(d(x)^{\mu_{i}}+\left|\xi_{1}\right|^{\gamma_{i}}+\left|\xi_{2}\right|^{\theta_{i}}\right),
$$

for a.e. $x \in \Omega$, for all $s_{i} \in \mathbb{R}$, and all $\xi_{i} \in \mathbb{R}^{N}$ such that

$$
\mu_{i}>-\frac{1}{r_{i}} \text { and } \max \left\{\gamma_{i}, \theta_{i}\right\}<\frac{p_{i}-1}{r_{i}} \text {, for } i=1,2 \text {. }
$$

The following result is a key point in the proof of Theorem 1.1.

Theorem 2.1. Assume $\left(\mathrm{H}_{h}\right)$ holds true. Then, there is a constant $k_{p}>0$, depending only on $p_{1}, p_{2}$ and $\Omega$, such that

$$
\|\nabla u\|_{\infty} \leq k_{p}\left\|h_{1}(\cdot, u, v, \nabla u, \nabla v)\right\|_{r_{1}}^{\frac{1}{p_{1}-1}}
$$

and

$$
\|\nabla v\|_{\infty} \leq k_{p}\left\|h_{2}(\cdot, u, v, \nabla u, \nabla v)\right\|_{r_{2}}^{\frac{1}{p_{2}-1}}
$$

Moreover, there are constants $R>0$ and $\sigma \in(0,1)$ such that all solutions $(u, v) \in W_{0}^{1, p_{1}}(\Omega) \times W_{0}^{1, p_{2}}(\Omega)$ of problem $\left(\mathrm{P}_{h}\right)$ belong to $C_{0}^{1, \sigma}(\bar{\Omega}) \times C_{0}^{1, \sigma}(\bar{\Omega})$ and satisfy the estimate

$$
\|u\|_{C^{1, \sigma}(\bar{\Omega})},\|v\|_{C^{1, \sigma}(\bar{\Omega})}<R
$$

Proof. Let $(u, v) \in W_{0}^{1, p_{1}}(\Omega) \times W_{0}^{1, p_{2}}(\Omega)$ be a solution of $\left(\mathrm{P}_{h}\right)$. Multiplying the first equation in $\left(\mathrm{P}_{h}\right)$ by $u$, integrating over $\Omega$ and using $\left(\mathrm{H}_{h}\right)$, one gets

$$
\begin{aligned}
& \int_{\Omega}|\nabla u|^{p_{1}} \mathrm{~d} x=\int_{\Omega} h_{1}(x, u, v, \nabla u, \nabla v) u \mathrm{~d} x \\
& \leq M \int_{\Omega}\left(d(x)^{\mu_{1}}+|\nabla u|^{\gamma_{1}}+|\nabla v|^{\theta_{1}}\right) u \mathrm{~d} x .
\end{aligned}
$$

Since $\mu_{1}>-1$, by Hardy-Sobolev inequality (see, e.g., [1, Lemma 2.3]), there exists a positive constant $C_{1}$ such that

$$
\int_{\Omega} d(x)^{\mu_{1}} u \mathrm{~d} x \leq C_{1}\|\nabla u\|_{p_{1}} .
$$


Using (2.2), Young inequality and (2.1) imply that

$$
\begin{aligned}
& \int_{\Omega}|\nabla u|^{\gamma_{1}} u \mathrm{~d} x \leq \varepsilon \int_{\Omega}|u|^{p_{1}} \mathrm{~d} x+c_{\varepsilon} \int_{\Omega}|\nabla u|^{\gamma_{1} p_{1}^{\prime}} \mathrm{d} x \\
& \leq \varepsilon\|\nabla u\|_{p_{1}}^{p_{1}}+c_{\varepsilon}\|\nabla u\|_{p_{1}}^{\gamma_{1} p_{1}^{\prime}}, \\
& \int_{\Omega}|\nabla v|^{\theta_{1}} u \mathrm{~d} x \leq \varepsilon \int_{\Omega}|u|^{p_{1}} \mathrm{~d} x+c_{\varepsilon} \int_{\Omega}|\nabla v|^{\theta_{1} p_{1}^{\prime}} \mathrm{d} x \\
& \leq \varepsilon\|\nabla u\|_{p_{1}}^{p_{1}}+c_{\varepsilon}\|\nabla v\|_{p_{2}}^{\theta_{1} p_{1}^{\prime}},
\end{aligned}
$$

for every $\varepsilon>0$ and with a certain constant $c_{\varepsilon}>0$ depending on $\varepsilon$.

Similarly, multiplying the second equation in $\left(\mathrm{P}_{h}\right)$ by $v$, integrating over $\Omega$ and repeating the previous argument leads to

$$
\begin{aligned}
& \int_{\Omega}|\nabla v|^{p_{2}} \mathrm{~d} x=\int_{\Omega} h_{2}(u, v, \nabla u, \nabla v) u \mathrm{~d} x \\
& \leq M \int_{\Omega}\left(d(x)^{\mu_{2}}+|\nabla u|^{\gamma_{2}}+|\nabla v|^{\theta_{2}}\right) v \mathrm{~d} x
\end{aligned}
$$

with

$$
\begin{gathered}
\int_{\Omega} d(x)^{\mu_{2}} v \mathrm{~d} x \leq C_{2}\|\nabla v\|_{p_{2}}, \\
\int_{\Omega}|\nabla v|^{\theta_{2}} v \mathrm{~d} x \leq \varepsilon\|\nabla v\|_{p_{2}}^{p_{2}}+c_{\varepsilon}^{\prime}\|\nabla v\|_{p_{2}}^{\theta_{2} p_{2}^{\prime}}, \\
\int_{\Omega}|\nabla u|^{\gamma_{2}} v \mathrm{~d} x \leq \varepsilon\|\nabla v\|_{p_{2}}^{p_{2}}+c_{\varepsilon}^{\prime}\|\nabla u\|_{p_{1}}^{\gamma_{2} p_{2}^{\prime}} .
\end{gathered}
$$

for $\varepsilon>0$ and a constant $c_{\varepsilon}^{\prime}>0$. On the other hand, since $r_{i}>N>\frac{p_{i}}{p_{j}}$, for $i, j=1,2, i \neq j$, it follows, from (2.2), that

$$
\max _{i=1,2}\left(\gamma_{i} p_{i}^{\prime}\right)<p_{1} \text { and } \max _{i=1,2}\left(\theta_{i} p_{i}^{\prime}\right)<p_{2}
$$

Then, gathering (2.4)-(2.11) together, on account of (2.12) and by taking $\varepsilon$ small, there is a constant $c_{0}>0$, independent of the solution $(u, v)$, such that

$$
\|\nabla u\|_{p_{1}},\|\nabla v\|_{p_{2}} \leq c_{0}
$$

Combining the last estimate with (2.5), through the growth condition $\left(\mathrm{H}_{h}\right)$, it follows that

$$
\left\|h_{i}(u, v, \nabla u, \nabla v)\right\|_{r_{i}} \leq c_{1},
$$

with a constant $c_{1}>0$ independent of the solution $(u, v)$. At this point we make use of the assumption that $r_{i}>N$, which enables us to refer to the gradient bound in [7, Theorem 3.1; Remark 3.3]. Consequently, due to (2.14), we infer that

$$
\|\nabla u\|_{\infty} \leq k_{p}\left\|h_{1}(\cdot, u, v, \nabla u, \nabla v)\right\|_{r_{1}}^{\frac{1}{p_{1}-1}}
$$

and

$$
\|\nabla v\|_{\infty} \leq k_{p}\left\|h_{2}(\cdot, u, v, \nabla u, \nabla v)\right\|_{r_{2}}^{\frac{1}{p_{2}-1}}
$$


where the constant $k_{p}>0$ depends only on $p_{1}, p_{2}$ and $\Omega$. On the other hand, by $\left(\mathrm{H}_{h}\right)$ and (2.14) $-(2.16)$ we have

$$
\begin{aligned}
& \left|h_{i}(x, u, v, \nabla u, \nabla v)\right| \leq M\left(d(x)^{\mu_{i}}+|\nabla u|^{\gamma_{i}}+|\nabla v|^{\theta_{i}}\right) \\
& \leq M\left(d(x)^{\mu_{i}}+\|\nabla u\|_{\infty}^{\gamma_{i}}+\|\nabla v\|_{\infty}^{\theta_{i}}\right) \\
& \leq M\left(d(x)^{\mu_{i}}+\left(k_{p} c_{1}^{\frac{1}{p_{1}-1}}\right)^{\gamma_{i}}+\left(k_{p} c_{1}^{\frac{1}{p_{2}-1}}\right)^{\theta_{i}}\right) \\
& \leq \hat{M}\left(d(x)^{\mu_{i}}+1\right) \text { in } \Omega,
\end{aligned}
$$

for some constant $\hat{M}>0$ independent of $u$ and $v$. Thus, since $\mu_{i}>-1$, the nonlinear regularity up to the boundary in [12, Lemma 3.1] applies, showing that the solution $(u, v)$ is bounded in $C_{0}^{1, \sigma}(\bar{\Omega}) \times C_{0}^{1, \sigma}(\bar{\Omega})$ for certain $\sigma \in(0,1)$. This ends the proof.

In the scalar case of $\left(\mathrm{P}_{h}\right)$, the previous regularity Theorem 2.1 establishes an extension of [12, Lemma 3.1] to singular problems involving convection terms. It is formulated as follows.

Corollary 2.2. For $1<p \leq N$ and $M>0$, let $h: \Omega \times \mathbb{R} \times \mathbb{R}^{N} \rightarrow \mathbb{R}$ be a Carathéodory function satisfying

$$
|h(x, s, \xi)| \leq M\left(d(x)^{\mu}+|\xi|^{\gamma}\right),
$$

for a.e. $x \in \Omega$, for all $s \in \mathbb{R}$, and all $\xi \in \mathbb{R}^{N}$ with

$$
r>N \text { and }-\frac{1}{r}<\mu<0 \leq \gamma<\frac{p-1}{r} .
$$

Then, there are constants $R>0$ and $\sigma \in(0,1)$ such that all solutions $u \in W_{0}^{1, p}(\Omega)$ of Dirichlet problem

$$
\left\{\begin{array}{l}
-\Delta_{p} u=h(x, u, \nabla u) \text { in } \Omega \\
u=0 \quad \text { on } \partial \Omega,
\end{array}\right.
$$

belong to $C_{0}^{1, \sigma}(\bar{\Omega}) \times C_{0}^{1, \sigma}(\bar{\Omega})$ and satisfy the estimate $\|u\|_{C^{1, \sigma}(\bar{\Omega})}<R$. Moreover, there is a constant $k_{p}>0$, depending only on $p$ and $\Omega$, such that

$$
\|\nabla u\|_{\infty} \leq k_{p}\|h(\cdot, u, \nabla u)\|_{r}^{\frac{1}{p-1}} .
$$

\section{COMPARISON PROPERTIES}

Let $y_{i}, z_{i} \in C^{1}(\bar{\Omega})$ satisfy

$$
-\Delta_{p_{i}} y_{i}(x)=1+d(x)^{\alpha_{i}+\beta_{i}} \text { in } \Omega, y_{i}(x)=0 \text { on } \partial \Omega,
$$

and

$$
-\Delta_{p_{i}} z_{i}(x)=\left\{\begin{array}{ll}
d(x)^{\alpha_{i}+\beta_{i}} & \text { in } \Omega \backslash \bar{\Omega}_{\delta} \\
-1 & \text { in } \Omega_{\delta}
\end{array}, z_{i}(x)=0 \text { on } \partial \Omega\right.
$$

where $\Omega_{\delta}:=\{x \in \Omega: d(x)<\delta\}$, with a fixed $\delta>0$ sufficiently small, and the exponents $\alpha_{i}, \beta_{i}$ satisfy

$$
-1<\alpha_{i}+\beta_{i}<p_{i}-1, \text { for } i=1,2 .
$$


Hardy-Sobolev inequality (see, e.g., [1, Lemma 2.3]) guarantees that the

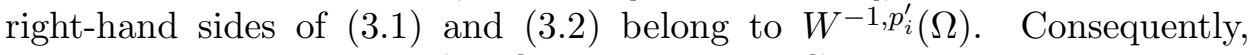
Minty-Browder Theorem (see [4, Theorem V.15]) ensures the existence of unique $y_{i}$ and $z_{i}$ in (3.2) and (3.1).

Lemma 3.1. There are positive constants $c_{0}$ and $c_{1}$ such that

$$
c_{0} d(x) \leq z_{i}(x) \leq y_{i}(x) \leq c_{1} d(x) \text { for all } x \in \Omega, i=1,2 .
$$

Proof. By (3.1) and (3.2), it is readily seen that $z_{i}(x) \leq y_{i}(x)$ for all $x \in \Omega$, for $i=1,2$. Moreover, the Strong Maximum Principle together with [12, Corollary 3.1] entail $c_{0} d(x) \leq z_{i}(x)$ in $\Omega$, for $\delta>0$ sufficiently small in (3.2). Thus, the proof is completed by showing the last inequality in (3.4). To this end, let consider the unique solution $\hat{w}_{i} \in C_{0}^{1, \tau}(\bar{\Omega})$ homogeneous Dirichlet problem

$$
-\Delta_{p_{i}} \hat{w}_{i}=1 \text { in } \Omega, \hat{w}_{i}=0 \text { on } \partial \Omega
$$

that satisfies

$$
\left\|\nabla \hat{w}_{i}\right\|_{\infty} \leq \hat{L}, \text { for certain constant } \hat{L}>0, i=1,2 .
$$

Since $\partial \Omega$ is smooth, we can find $\delta>0$ and $\Pi \in C^{1}\left(\Omega_{\delta}, \partial \Omega\right)$ fulfilling

$$
\left.\left.d(x)=|x-\Pi(x)|, \quad \frac{x-\Pi(x)}{|x-\Pi(x)|}=-\eta(\Pi(x)), \quad\right] \Pi(x), x\right] \subseteq \Omega, \quad x \in \Omega_{\delta},
$$

where $\eta(x)$ indicates the outward unit normal vector to $\partial \Omega$ at its point $x$. Thus, the Mean Value Theorem, when combined with (3.6), lead to

$$
\left|\hat{w}_{i}(x)\right|=\left|\hat{w}_{i}(x)-\hat{w}_{i}(\Pi(x))\right| \leq \hat{L}|x-\Pi(x)|=\hat{L} d(x) \quad \forall x \in \Omega_{\delta} .
$$

Define

$$
L:=\max \left\{\hat{L}, \max _{\Omega \backslash \Omega_{\delta}} \frac{\hat{w}_{i}}{d}, i=1,2\right\} .
$$

On account of (3.8), one evidently has

$$
\hat{w}_{i} \leq L d(x) \text { in } \Omega \text {. }
$$

Let us ssume that $\alpha_{i}+\beta_{i} \geq 0$ in (3.3). Then, there is a constant $\hat{c}>0$ such that $1+d(x)^{\alpha_{i}+\beta_{i}} \leq \hat{c}$. From (3.5) and (3.1) one derives that

$$
-\Delta_{p_{i}}\left(\hat{c}^{\frac{-1}{p_{i}-1}} y_{i}\right) \leq-\Delta_{p_{i}} \hat{w}_{i} \text { in } \Omega,
$$

which, by (3.9) and the weak comparison principle (see [24, Lemma 3.1]), implies that $y_{i}(x) \leq c_{1} d(x)$ in $\Omega$, for certain constant $c_{1}$. Now, assume that $\alpha_{i}+\beta_{i}<0$ in (3.3) and let $w_{i} \in C^{1}(\bar{\Omega})$, be the unique weak solution of Dirichlet problem

$$
\left\{\begin{array}{ll}
-\Delta_{p_{i}} w_{i}=w_{i}^{-\gamma} & \text { in } \Omega \\
w_{i}>0 & \text { in } \Omega, \\
w_{i}=0 & \text { on } \partial \Omega
\end{array}, i=1,2\right.
$$


where

$$
\max _{i=1,2}\left|\alpha_{i}+\beta_{i}\right|<\gamma<1
$$

It is well known that

$$
c_{2} d(x) \leq w_{i}(x) \leq c_{3} d(x), i=1,2
$$

with positive constants $c_{2}, c_{3}$ (see [14]). Due to (3.11), one can find a constant $L>0$ such that

$$
\max _{i=1,2}\left(1+d(x)^{\alpha_{i}+\beta_{i}}\right) d(x)^{\gamma} \leq L \text { in } \bar{\Omega} .
$$

On account of (3.1), (3.13) and (3.12), it follows that

$$
\begin{aligned}
& -\Delta_{p_{i}} y_{i}(x)=1+d(x)^{\alpha_{i}+\beta_{i}} \leq L d(x)^{-\gamma} \\
& \leq L\left(c_{3} w_{i}\right)^{-\gamma}=-\Delta_{p_{i}}\left(\left(L c_{3}^{-\gamma}\right)^{\frac{1}{p_{i}-1}} w_{i}(x)\right) \text { in } \Omega .
\end{aligned}
$$

Then, the weak comparison principle (see [24, Lemma 3.1]) leads to

$$
y_{i}(x) \leq\left(L c_{3}^{-\gamma}\right)^{\frac{1}{p_{i}-1}} w_{i}(x) \leq L^{\frac{1}{p_{i}-1}} c_{3}^{1-\frac{\gamma}{p_{i}-1}} d(x) \text { in } \Omega .
$$

This ends the proof.

Set

$$
(\underline{u}, \underline{v})=C^{-1}\left(z_{1}, z_{2}\right) \quad \text { and } \quad(\bar{u}, \bar{v})=C\left(y_{1}, y_{2}\right)
$$

where $C>1$ is a constant. Obviously, $\underline{u} \leqslant \underline{v}$ and $\bar{u} \leqslant \bar{v}$ in $\bar{\Omega}$.

The following result allows us to achieve useful comparison properties.

Proposition 3.1. Assume (3.3) holds true and let $\gamma_{i}, \theta_{i} \geq 0$ be real constants such that

$$
\max \left\{\gamma_{i}, \theta_{i}\right\}<p_{i}-1, \quad \text { for } i=1,2 .
$$

Then, for $C>0$ sufficiently large in (3.14), it holds

$$
\begin{gathered}
-\Delta_{p_{1}} \underline{u} \leq m_{1} w_{1}^{\alpha_{1}} w_{2}^{\beta_{1}} \quad \text { in } \Omega, \\
-\Delta_{p_{2}} \underline{v} \leq m_{2} w_{1}^{\alpha_{2}} w_{2}^{\beta_{2}} \text { in } \Omega, \\
-\Delta_{p_{1}} \bar{u} \geq M_{1} w_{1}^{\alpha_{1}} w_{2}^{\beta_{1}}+2 C^{\max \left\{\gamma_{1}, \theta_{1}\right\}} \quad \text { in } \Omega
\end{gathered}
$$

and

$$
-\Delta_{p_{2}} \bar{v} \geq M_{2} w_{1}^{\alpha_{2}} w_{2}^{\beta_{2}}+2 C^{\max \left\{\gamma_{2}, \theta_{2}\right\}} \quad \text { in } \Omega,
$$

whenever $\left(w_{1}, w_{2}\right) \in[\underline{u}, \bar{u}] \times[\underline{v}, \bar{v}]$. 
Proof. On the basis of (3.14) and Lemma 3.1 , for all $\left(w_{1}, w_{2}\right) \in[\underline{u}, \bar{u}] \times[\underline{v}, \bar{v}]$, on has

$$
\begin{aligned}
w_{1}^{\alpha_{i}} w_{2}^{\beta_{i}} \geq & \begin{cases}\underline{u}^{\alpha_{i}} \underline{v}^{\beta_{i}} & \text { if } \alpha_{i}, \beta_{i}>0 \\
\bar{u}^{\alpha_{i}} \underline{v}^{\beta_{i}} & \text { if } \alpha_{i}<0<\beta_{i} \\
\underline{u}^{\alpha_{i}} \bar{v}^{\beta_{i}} & \text { if } \beta_{i}<0<\alpha_{i} \\
\bar{u}^{\alpha_{i}} \bar{v}^{\beta_{i}} & \text { if } \alpha_{i}, \beta_{i}<0\end{cases} \\
\geq & \begin{cases}\left(C^{-1} c_{0} d(x)\right)^{\alpha_{i}+\beta_{i}} & \text { if } \alpha_{i}, \beta_{i}>0 \\
\left(C c_{1}\right)^{\alpha_{i}}\left(C^{-1} c_{0}\right)^{\beta_{i}} d(x)^{\alpha_{i}+\beta_{i}} & \text { if } \alpha_{i}<0<\beta_{i} \\
\left(C^{-1} c_{0}\right)^{\alpha_{i}}\left(C c_{1}\right)^{\beta_{i}} d(x)^{\alpha_{i}+\beta_{i}} & \text { if } \beta_{i}<0<\alpha_{i} \\
\left(C c_{1}\right)^{\alpha_{i}+\beta_{i}} d(x)^{\alpha_{i}+\beta_{i}} & \text { if } \alpha_{i}, \beta_{i}<0\end{cases} \\
\geq \tilde{c}_{0} C^{-\left(\left|\alpha_{i}\right|+\left|\beta_{i}\right|\right)} d(x)^{\alpha_{i}+\beta_{i}} &
\end{aligned}
$$

and

$$
\begin{aligned}
w_{1}^{\alpha_{i}} w_{2}^{\beta_{i}} \leq & \begin{cases}\bar{u}^{\alpha_{i}} \bar{v}^{\beta_{i}} & \text { if } \alpha_{i}, \beta_{i}>0 \\
\underline{u}^{\alpha_{i}} \bar{v}^{\beta_{i}} & \text { if } \alpha_{i}<0<\beta_{i} \\
\bar{u}^{\alpha_{i}} \underline{v}^{\beta_{i}} & \text { if } \beta_{i}<0<\alpha_{i} \\
\underline{u}^{\alpha_{i}} \underline{v}^{\beta_{i}} & \text { if } \alpha_{i}, \beta_{i}<0\end{cases} \\
& \leq \begin{cases}\left(C c_{1} d(x)\right)^{\alpha_{i}+\beta_{i}} & \text { if } \alpha_{i}, \beta_{i}>0 \\
\left(C^{-1} c_{0}\right)^{\alpha_{i}}\left(C c_{1}\right)^{\beta_{i}} d(x)^{\alpha_{i}+\beta_{i}} & \text { if } \alpha_{i}<0<\beta_{i} \\
\left(C c_{1}\right)^{\alpha_{i}}\left(C^{-1} c_{0}\right)^{\beta_{i}} d(x)^{\alpha_{i}+\beta_{i}} & \text { if } \beta_{i}<0<\alpha_{i} \\
\left(C^{-1} c_{0}\right)^{\alpha_{i}}\left(C^{-1} c_{0}\right)^{\beta_{i}} d(x)^{\alpha_{i}+\beta_{i}} & \text { if } \alpha_{i}, \beta_{i}<0\end{cases} \\
& \leq \tilde{c}_{1} C^{\left|\alpha_{i}\right|+\left|\beta_{i}\right|} d(x)^{\alpha_{i}+\beta_{i}},
\end{aligned}
$$

where $\tilde{c}_{0}, \tilde{c}_{1}$ are positive constants depending on $c_{0}, c_{1}, \alpha_{i}, \beta_{i}$. From (3.14), (3.2), (3.1) and (3.15), one gets

$$
\begin{aligned}
& -\Delta_{p_{1}} \underline{u}=C^{-\left(p_{1}-1\right)} \begin{cases}d(x)^{\alpha_{1}+\beta_{1}} & \text { in } \Omega \backslash \bar{\Omega}_{\delta} \\
-1 & \text { in } \Omega_{\delta}\end{cases} \\
& \leq \tilde{c}_{0} m_{1} C^{-\left(\left|\alpha_{1}\right|+\left|\beta_{1}\right|\right)} d(x)^{\alpha_{1}+\beta_{1}} \text { in } \Omega, \\
& -\Delta_{p_{2}} \underline{v}=C^{-\left(p_{2}-1\right)} \begin{cases}d(x)^{\alpha_{2}+\beta_{2}} & \text { in } \Omega \backslash \bar{\Omega}_{\delta} \\
-1 & \text { in } \Omega_{\delta}\end{cases} \\
& \leq \tilde{c}_{0} m_{2} C^{-\left(\left|\alpha_{2}\right|+\left|\beta_{2}\right|\right)} d(x)^{\alpha_{2}+\beta_{2}} \text { in } \Omega, \\
& -\Delta_{p_{1}} \bar{u}=C^{p_{1}-1}\left(1+d(x)^{\alpha_{1}+\beta_{1}}\right) \\
& \geq \tilde{c}_{1} M_{1} C^{\left|\alpha_{1}\right|+\left|\beta_{1}\right|} d(x)^{\alpha_{1}+\beta_{1}}+2 C^{\max \left\{\gamma_{1}, \theta_{1}\right\}} \text { in } \Omega
\end{aligned}
$$

and

$$
\begin{aligned}
& -\Delta_{p_{2}} \bar{v}=C^{p_{2}-1}\left(1+d(x)^{\alpha_{2}+\beta_{2}}\right) \\
& \geq \tilde{c}_{1} M_{2} C^{\left|\alpha_{2}\right|+\left|\beta_{2}\right|} d(x)^{\alpha_{2}+\beta_{2}}+2 C^{\max \left\{\gamma_{2}, \theta_{2}\right\}} \text { in } \Omega,
\end{aligned}
$$

provided $C>1$ sufficiently large. Consequently, combining (3.16)-(3.21) the desired estimates follow. 


\section{Existence of SOlutions}

By using the functions in (3.14), let us introduce the set

$$
\mathcal{O}_{C}:=\left\{\begin{array}{c}
(\mathrm{u}, \mathrm{v}) \in C_{0}^{1}(\bar{\Omega})^{2}: \underline{u} \leqslant \mathrm{u} \leqslant \bar{u}, \underline{v} \leqslant \mathrm{v} \leqslant \bar{v} \text { in } \Omega \\
\text { and }\|\nabla \mathrm{u}\|_{\infty},\|\nabla \mathrm{v}\|_{\infty} \leqslant C
\end{array}\right\},
$$

which is closed, bounded and convex in $C_{0}^{1}(\bar{\Omega}) \times C_{0}^{1}(\bar{\Omega})$. Define the operator $\mathcal{T}: \mathcal{O}_{C} \rightarrow C_{0}^{1}(\bar{\Omega}) \times C_{0}^{1}(\bar{\Omega})$ by $\mathcal{T}\left(w_{1}, w_{2}\right)=(u, v)$ for all $\left(w_{1}, w_{2}\right) \in \mathcal{O}_{C}$, where $(u, v)$ is required to satisfy

$$
\left(\mathrm{P}_{w}\right) \quad \begin{cases}-\Delta_{p_{1}} u=f_{1}\left(x, w_{1}, w_{2}, \nabla w_{1}, \nabla w_{2}\right) & \text { in } \Omega \\ -\Delta_{p_{2}} v=f_{2}\left(x, w_{1}, w_{2}, \nabla w_{1}, \nabla w_{2}\right) & \text { in } \Omega \\ u, v=0 \quad \text { on } \partial \Omega . & \end{cases}
$$

In view of the definition $\mathcal{O}_{C}$ and by $\left(\mathrm{H}_{f}\right)$, we have

$$
\begin{aligned}
& m_{i} w_{1}^{\alpha_{i}} w_{2}^{\beta_{i}} \leq f_{i}\left(x, w_{1}, w_{2}, \nabla w_{1}, \nabla w_{2}\right) \\
& \leq M_{i} w_{1}^{\alpha_{i}} w_{2}^{\beta_{i}}+\left|\nabla w_{1}\right|^{\gamma_{i}}+\left|\nabla w_{2}\right|^{\theta_{i}} \\
& \leq M_{i} w_{1}^{\alpha_{i}} w_{2}^{\beta_{i}}+\left\|\nabla w_{1}\right\|_{\infty}^{\gamma_{i}}+\left\|\nabla w_{2}\right\|_{\infty}^{\theta_{i}} \\
& \leq M_{i} w_{1}^{\alpha_{i}} w_{2}^{\beta_{i}}+2 C^{\max \left\{\gamma_{i}, \theta_{i}\right\}} \text { in } \Omega, \text { for } i=1,2 .
\end{aligned}
$$

Hence, recalling (3.17) we achieve

$$
\begin{aligned}
& f_{i}\left(x, w_{1}, w_{2}, \nabla w_{1}, \nabla w_{2}\right) \\
& \leq M_{i} \tilde{c}_{1} C^{\left|\alpha_{i}\right|+\left|\beta_{i}\right|} d(x)^{\alpha_{i}+\beta_{i}}+2 C^{\max \left\{\gamma_{i}, \theta_{i}\right\}} \text { in } \Omega, \text { for } i=1,2 .
\end{aligned}
$$

Then, since $\alpha_{i}+\beta_{i}>-1$, Hardy-Sobolev inequality (see, e.g., 1, Lemma

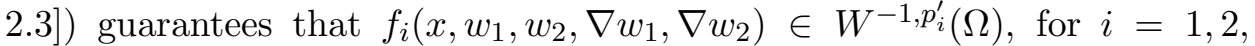
while the unique solvability of $(u, v)$ in $\left(\mathrm{P}_{w}\right)$ is readily derived from Minty Browder's Theorem (see, e.g., [4]). Thus, $\mathcal{T}$ is well defined. Moreover, the regularity theory up to the boundary in [19] or [12], depending on whether the sign of $\alpha_{i}+\beta_{i}$ is positive $\left(\alpha_{i}+\beta_{i} \geq 0\right)$ or negative $\left(\alpha_{i}+\beta_{i} \in(-1,0)\right)$, respectively, yields $(u, v) \in C_{0}^{1, \sigma}(\bar{\Omega}) \times C_{0}^{1, \sigma}(\bar{\Omega})$ and there is a constant $R>0$ and $\sigma \in(0,1)$ such that it holds

$$
\|u\|_{C^{1, \sigma}(\bar{\Omega})},\|v\|_{C^{1, \sigma}(\bar{\Omega})}<R .
$$

It is worth noting that the weak solutions of problem $\left(\mathrm{P}_{w}\right)$ coincide with the fixed points of the operator $\mathcal{T}$. To reach the desired conclusion, we shall apply Schauder's fixed point theorem (see, for example, [25, p. 57]).

We claim that the map $\mathcal{T}: \mathcal{O}_{C} \rightarrow C_{0}^{1}(\bar{\Omega}) \times C_{0}^{1}(\bar{\Omega})$ is continuous and compact. Let $\left(w_{1, n}, w_{2, n}\right) \in \mathcal{O}_{C}$ such that $\left(w_{1, n}, w_{2, n}\right) \longrightarrow\left(w_{1}, w_{2}\right)$ in $C_{0}^{1, \sigma}(\bar{\Omega}) \times C_{0}^{1, \sigma}(\bar{\Omega})$. Setting $\mathcal{T}\left(w_{1, n}, w_{2, n}\right)=\left(u_{n}, v_{n}\right)$ reads as

$$
\left\{\begin{array}{l}
\left\langle-\Delta_{p_{1}} u_{n}, \varphi\right\rangle=\int_{\Omega} f_{1}\left(w_{1, n}, w_{2, n} \nabla w_{1, n}, \nabla w_{2, n}\right) \varphi \mathrm{d} x \\
\left\langle-\Delta_{p_{2}} v_{n}, \psi\right\rangle=\int_{\Omega} f_{2}\left(w_{1, n}, w_{2, n} \nabla w_{1, n}, \nabla w_{2, n}\right) \psi \mathrm{d} x
\end{array}\right.
$$

for all $(\varphi, \psi) \in W_{0}^{1, p_{1}}(\Omega) \times W_{0}^{1, p_{2}}(\Omega)$. Acting in (4.4) with $(\varphi, \psi)=\left(u_{n}-\right.$ $\left.u, v_{n}-v\right)$, (4.2) and (3.3) allow to apply Lebesgue's dominated convergence 
theorem to deduce that

$$
\lim _{n \longrightarrow \infty}\left\langle-\Delta_{p_{1}} u_{n}, u_{n}-u\right\rangle=\lim _{n \rightarrow \infty}\left\langle-\Delta_{p_{2}} v_{n}, v_{n}-v\right\rangle=0 .
$$

Here the integrability of the functions $d(x)^{\alpha_{1}+\beta_{1}}\left(u_{n}-u\right)$ and $d(x)^{\alpha_{2}+\beta_{2}}\left(v_{n}-\right.$ $v)$, which is known by invoking the Hardy-Sobolev inequality under assumption (3.3), is essential. At this point, the $S_{+}$-property of $-\Delta_{p_{i}}$ on $W_{0}^{1, p_{i}}(\Omega)$ ensures that

$$
\left(u_{n}, v_{n}\right) \longrightarrow(u, v) \text { in } W_{0}^{1, p_{1}}(\Omega) \times W_{0}^{1, p_{2}}(\Omega) .
$$

Hence, passing to the limit in (4.4) leads to $(u, v)=\mathcal{T}\left(w_{1}, w_{2}\right)$. On the other hand, from (4.3) we know that the sequence $\left\{\left(u_{n}, v_{n}\right)\right\}$ is bounded in $C_{0}^{1, \sigma}(\bar{\Omega}) \times C_{0}^{1, \sigma}(\bar{\Omega})$ for certain $\sigma \in(0,1)$. Since the embedding $C_{0}^{1, \sigma}(\bar{\Omega}) \subset$ $C_{0}^{1}(\bar{\Omega})$ is compact and taking into account (4.5), it turns out that along a relabeled subsequence one has that $\left(u_{n}, v_{n}\right) \longrightarrow(u, v)$ in $C_{0}^{1}(\bar{\Omega}) \times C_{0}^{1}(\bar{\Omega})$. Therefore, $\mathcal{T}$ is continuous. Again, through (4.3), it follows that $\mathcal{T}\left(\mathcal{O}_{C}\right)$ is bounded in $C_{0}^{1, \sigma}(\bar{\Omega}) \times C_{0}^{1, \sigma}(\bar{\Omega})$. Then, invoking the compactness of the embedding $C_{0}^{1, \sigma}(\bar{\Omega}) \subset C_{0}^{1}(\bar{\Omega})$, we conclude that $\mathcal{T}\left(\mathcal{O}_{C}\right)$ is a relatively compact subset of $C_{0}^{1}(\bar{\Omega}) \times C_{0}^{1}(\bar{\Omega})$. This proves the claim.

We are left to show that $\mathcal{O}_{C}$ is invariant under $\mathcal{T}$. Let $\left(w_{1}, w_{2}\right) \in \mathcal{O}_{C}$ and denote $(u, v)=\mathcal{T}\left(w_{1}, w_{2}\right)$. Using $\left(\mathrm{P}_{w}\right)$ and combining Proposition 3.1 with (4.1), the weak comparison principle yields

$$
\underline{u} \leq u \leq \bar{u} \text { and } \underline{v} \leq v \leq \bar{v} \text { in } \Omega .
$$

On the other hand, on the basis of (4.2) and (1.2), Theorem 2.1 entails

$$
\|\nabla u\|_{\infty},\|\nabla v\|_{\infty} \leq k_{p} \max _{i=1,2}\left\|f_{i}(\cdot, u, v, \nabla u, \nabla v)\right\|_{r_{i}}^{\frac{1}{p_{i}-1}},
$$

for some positive constant $k_{p}$ depending only on $p_{i}$ and $\Omega$. Exploiting once again (4.2) one has

$$
\begin{aligned}
& || f_{i}\left(\cdot, w_{1}, w_{2}, \nabla w_{1}, \nabla w_{2}\right) \|_{r_{i}} \\
& \leq M_{i} \tilde{c}_{1} C^{\left|\alpha_{i}\right|+\left|\beta_{i}\right|}\left(\int_{\Omega} d(x)^{r_{i}\left(\alpha_{i}+\beta_{i}\right)} \mathrm{d} x\right)^{1 / r_{i}}+2|\Omega| C^{\max \left\{\gamma_{i}, \theta_{i}\right\}} .
\end{aligned}
$$

Bearing in mind (1.2), [18, Lemma] applies, showing that the integral term $\left(\int_{\Omega} d(x)^{r_{i}\left(\alpha_{i}+\beta_{i}\right)} \mathrm{d} x\right)^{1 / r_{i}}$ is bounded by some constant $\mu>0$. At this point, from (4.8) and (1.2), we have

$$
\begin{aligned}
& || f_{i}\left(\cdot, w_{1}, w_{2}, \nabla w_{1}, \nabla w_{2}\right) \|_{r_{i}} \\
& \leq M_{i} \tilde{c}_{1} C^{\left|\alpha_{i}\right|+\left|\beta_{i}\right|} \mu+2|\Omega| C^{\max \left\{\gamma_{i}, \theta_{i}\right\}} \leq\left(k_{p}^{-1} C\right)^{p_{i}-1},
\end{aligned}
$$

provided $C>1$ large enough. Hence, owing to (4.7) we conclude that

$$
\|\nabla u\|_{\infty},\|\nabla v\|_{\infty} \leq C .
$$

Gathering (4.6) and (4.10) enable us to infer that $(u, v) \in \mathcal{O}_{C}$, thereby the inclusion $\mathcal{T}\left(\mathcal{O}_{C}\right) \subset \mathcal{O}_{C}$ holds true. 
We are thus in a position to apply Schauder fixed point theorem to the map $\mathcal{T}: \mathcal{O}_{C} \rightarrow \mathcal{O}_{C}$, which establishes the existence of $(u, v) \in \mathcal{O}_{C}$ satisfying $(u, v)=\mathcal{T}(u, v)$. In view of the definition of $\mathcal{T}$, it turns out that $(u, v)$ is a (positive) smooth solution of problem $(\mathrm{P})$. This ends the proof of Theorem 1.1 .

Acknowledgement 1 . The authors were supported by the Directorate-General of Scientific Research and Technological Development (DGRSDT).

\section{REFERENCES}

[1] C.O. Alves \& F.J.S.A. Correa, On the existence of positive solution for a class of singular systems involving quasilinear operators, Appl. Math. Comput. 185 (2007), 727-736.

[2] C.O. Alves, P.C. Carrião \& L.F.O. Faria, Existence of solutions to singular elliptic equations with convection terms via the Galerkin method, Electron. J. Diff. Eqts. 12 (2010), 1-12.

[3] C. O. Alves \& A. Moussaoui, Existence of solutions for a class of singular elliptic systems with convection term, Asymptot. Anal. 90 (2014), 237-248.

[4] H. Brezis, Analyse Fonctionnelle Theorie et Applications. Masson, Paris (1983).

[5] H. Bueno \& G. Ercole, A quasilinear problem with fast growing gradient, Appl. Math. Lett. 26 (2013), 520-523.

[6] P. Candito, R. Livrea \& A. Moussaoui, Singular quasilinear elliptic systems involving gradient terms, Nonl. Anal.: Real World Appl. 55 (2020), 103142.

[7] A. Cianchi \& V. Maz'ya, Global gradient estimates in elliptic problems under minimal data and domain regularity, Commun. Pure Appl. Anal. 14 (2015), 285-311.

[8] J.I. Diaz \& L. Tello, On a climate model with a dynamic nonlinear diffusive boundary condition, Disc. Cont. Dyn. Syst. Serie S 1 (2) 2008, 253-262.

[9] H. Didi \& A. Moussaoui, Multiple positive solutions for a class of quasilinear singular elliptic systems, Rend. Circ. Mat. Palermo, II. Ser 69 (2020), 977-994.

[10] H. Didi, B. Khodja \& A. Moussaoui, Singular Quasilinear Elliptic Systems With (super-) Homogeneous Condition, J. Sibe. Fede. Univ. Math. Phys. 13(2 ) (2020), 1-9.

[11] S. El Manouni, K. Perera \& R. Shivaji, On singular quasimonotone (p,q)-Laplacian systems, Proc. Roy. Soc. Edinburgh Sect. A 142 (2012), 585-594.

[12] D. D. Hai, On a class of singular p-Laplacian boundary value problems, J. Math. Anal. Appl. 383 (2011), 619-626.

[13] M. Ghergu, Lane-Emden systems with negative exponents, J. Funct. Anal. 258 (2010), 3295-3318.

[14] J. Giacomoni, I. Schindler \& P. Takac, Sobolev versus Hölder local minimizers and existence of multiple solutions for a singular quasilinear equation, Ann. Sc. Norm. Super. Pisa Cl. Sci. (5) 6 (2007), 117-158.

[15] M. Kaviany, Principles of Convective Heat Transfer. Springer-Verlag New York, 2001.

[16] B. Khodja \& A. Moussaoui, Positive solutions for infinite semipositone/positone quasilinear elliptic systems with singular and superlinear terms, Diff. Eqts. App. 8(4) (2016), 535-546.

[17] E.H. Kim, Singular Gierer-Meinhardt systems of elliptic boundary value problems, J. Math. Anal. Appl. 308 (2005), 1-10.

[18] A. C. Lazer \& P. J. Mckenna, On a singular nonlinear elliptic boundary-value problem, Proc. American Math. Soc. 3 (111) 1991, 721-730.

[19] G. M. Lieberman, Boundary regularity for solutions of degenerate elliptic equations, Nonlinear Anal. 12 (1988), 1203-1219. 
[20] D. Motreanu \& A. Moussaoui, An existence result for a class of quasilinear singular competitive elliptic systems, Appl. Math. Lett. 38 (2014), 33-37.

[21] D. Motreanu \& A. Moussaoui, A quasilinear singular elliptic system without cooperative structure, Acta Math. Sci. 34 (B) (2014), 905-916.

[22] D. Motreanu \& A. Moussaoui, Existence and boundedness of solutions for a singular cooperative quasilinear elliptic system, Complex Var. Elliptic Equ. 59 (2014), 285-296.

[23] D. Motreanu, A. Moussaoui \& Z. Zhang, Positive solutions for singular elliptic systems with convection term, J. Fixed Point Theory Appl. 19 (3) (2017), 2165-2175.

[24] P. Tolksdorf, On the Dirichlet problem for quasilinear equations in domains with conical boundary points, Comm. PDE. 8 (1983), 773-817.

[25] E. Zeidler, Nonlinear functional analysis and its applications. I. Fixed-point theorems. Springer-Verlag New York, 1986.

H. Dellouche, Applied Mathematics Laboratory (LMA), Faculty of Exact Sciences, A. Mira Bejaia University, Targa Ouzemour, 06000 Bejaia, Algeria

A. Moussaoui, Applied Mathematics Laboratory (LMA), Faculty of Exact Sciences, and Biology departement, Faculty of Natural \& Life Sciences, A. Mira Bejaia University, Targa Ouzemour, 06000 Bejaia, Algeria 